\title{
Bandwidth Segmentation Method for Ultra-High Resolution SAR Imaging
}

\author{
Xin Wang, Qingyong Gong, Rui Jiang, Daiyin Zhu \\ Nanjing University of Posts \& Telecommunications, Nanjing, China \\ wangx@njupt.edu.cn,j_ray@njupt.edu.cn,gqy_lover@njupt.edu.cn, zhudy@nuaa.edu.cn
}

\begin{abstract}
A new methodology to process received signal in $\mathrm{X}$ band ultra-high resolution synthetic aperture radar (SAR) is presented in this paper. The received wideband signal is decomposed into sub bandwidth signals firstly, which are compressed by the chirp scaling algorithm (CSA) and then combined in 2-D frequency domain to obtain the final image. Compared with original CSA, this bandwidth segmentation method improves SAR image quality and extends the focused scene size effectively. Point target simulation validates the algorithm.
\end{abstract}

Index Terms-ultra-high resolution SAR; bandwidth segmentation method; chirp scaling algorithm; 2-D frequency domain combination

\section{INTRODUCTION}

Problems will arise when utilizing existing approximate frequency domain imaging algorithms to process $\mathrm{X}$ band ultrahigh resolution synthetic aperture radar $(\mathrm{SAR})^{[1]-[7]}$ data directly. As the phase error in approximate frequency algorithms ${ }^{[8]-[11]}$ increases in ultra-high resolution cases, the focal quality of SAR image suffer significant degradation. The compensation for high order phase error terms ${ }^{[12]-[13]}$ can be applied to improve the accuracy of these algorithms to some extent but can not resolve this problem in nature.

To solve the above problems, this paper proposes a novel bandwidth segmentation method to for $\mathrm{X}$ band ultra-high resolution SAR imaging. In this methodology, the decomposed sub bandwidth signals are compressed first by CSA ${ }^{[10]}$ and then combined in 2-D frequency domain to obtain the final image. Performance analysis and simulation indicate that this novel method can improve focal quality of SAR image effectively, and compensate for most of the phase differences between sub pulses in one burst.

In the next section, SAR signal model is discussed and established. The processing flow of the bandwidth segmentation method is then described in Section III. Finally, point target simulation in Section IV validates this methodology.

\section{SIGNAL MODEL}

Radar transmits stepped chirps with modulation rate, and bandwidth . The received signal from a PT in the scene can be denoted as

$$
\begin{aligned}
& S_{r}(\tau, t, k)=w_{r}\left[\tau-\frac{2 R(t)}{c}\right] \cdot w_{a}\left(t-t_{c}\right) \\
& \cdot \exp \left\{j \pi \gamma\left[\tau-\frac{2 R(t)}{c}\right]^{2}\right\} \cdot \exp \left[-j 4 \pi f_{c} \frac{R(t)}{c}\right]
\end{aligned}
$$

where the center frequency is $f_{c}, \tau$ is the fast time while $t$ is the slow time centered at $t_{c}, R(t)$ denotes the instantaneous slant range from radar $\mathrm{APC}$ to the $\mathrm{PT}, w_{r}(\tau)$ is the range envelope while $w_{a}(t)$ is the azimuth envelope determined by the composite antenna pattern.

\section{BANDWIDTH SEGMENTATION METHOD}

As indicated in Section I, when using CSA to focus the received chirps in ultra-high resolution $S A R$, the focal quality of image will suffer significant degradation as a result of the increased phase error in CSA. To solve the above problems, the received chirp signals are processed via a novel methodology in this section, where the detailed procedure will be described in the following.

A. Decomposition of the received signal

Performing decomposition on the received signal in SAR, we can obtain the sub band data. Since the phase error in CSA is proportional to the bandwidth of processed signal, compression of decomposed sub band data can improve image quality effectively. The decomposed sub data can be expressed as

$$
\begin{aligned}
S_{r s}\left(\tau, t_{k}, k\right)= & w_{r}\left[\tau-\frac{2 R\left(t_{k}\right)}{c}\right] w_{a}\left(t_{k}-t_{c k}\right) \\
& \exp \left\{j \pi \gamma\left[\tau-\frac{2 R\left(t_{k}\right)}{c}\right]^{2}\right\} \\
& \exp \left[-j 4 \pi f_{c k} \frac{R\left(t_{k}\right)}{c}\right]
\end{aligned}
$$

where, $t_{k}$ is the azimuth time of sub data, which is centered at $t_{c k}$ with the sampling rate $\frac{P R F}{N}, f_{c k}$ is the center 
frequency of sub data, the parameter $k$ corresponds to the $k$ th sub data.

B. Sub Data Compression

Performing azimuth Fourier transform on (2) and compressing the sub bandwidth data via CSA, we can obtain

$$
\begin{aligned}
S_{2}\left(\tau, f_{a}, k\right)= & \sin c\left(\tau-2 \frac{R_{c}}{c}\right) \cdot w_{a}\left(f_{a k}\right) \\
& \cdot \exp \left(j 2 \pi f_{a k} \frac{k}{P R F}\right)
\end{aligned}
$$

where the filters are calculated according to the center frequency of sub data. $f_{r}$ is range frequency.

C. 2-D Frequency Domain Combination

Images with coarse resolution can be produced by performing azimuth IFFT on (3).To acquire the ultra-high resolution image, pulse compressed sub data need to be combined. Transforming the sub data into 2-D frequency domain, (3) becomes

$$
\begin{aligned}
S_{3}\left(f_{r}, f_{a}, k\right)= & w_{r}\left(f_{r}\right) \cdot w_{a}\left(f_{a}\right) \cdot \exp \left(-j 4 \pi \frac{f_{r}}{c} R_{c}\right) \\
& \cdot \exp \left(j 2 \pi f_{a} \frac{k}{P R F}\right)
\end{aligned}
$$

The synthesized signal can then be derived as

$$
\begin{aligned}
S_{c}\left(f_{r_{-} \text {new }}, f_{a}\right) & =\sum_{k=0}^{N-1}\left\{S_{3}\left(f_{r}+f_{c k}-\Delta f_{k}, f_{a}, k\right)\right. \\
& \left.\cdot \exp \left(-j 2 \pi f_{a} \frac{k}{P R F}\right)\right\}
\end{aligned},
$$

where

$$
\Delta f_{k}=\left(k+\frac{1}{2}-\frac{N}{2}\right) \cdot \Delta f, k=0,1, \ldots . N-1,
$$

$f_{r_{-} \text {new }}$ is the new range frequency after combination. In (5), the phase term $\left(-2 \pi f_{a} \frac{k}{P R F}\right)$ can compensate for most of the phase differences between neighboring pulses in one burst. Performing 2-D IFFT on (5), the final image can be obtained.

In conclusion, the detailed processing flow can be depicted in Fig.2.

\section{POINT TARGET SIMULATION}

In this section, PT simulation will be carried out to prove the validity of bandwidth segmentation method, where the parameters are listed in Table II. 15 targets in the ground are applied in the simulation. As depicted in Fig. 3, the intervals between neighboring targets along range and azimuth dimensions are $375 \mathrm{~m}$ and $150 \mathrm{~m}$, respectively.

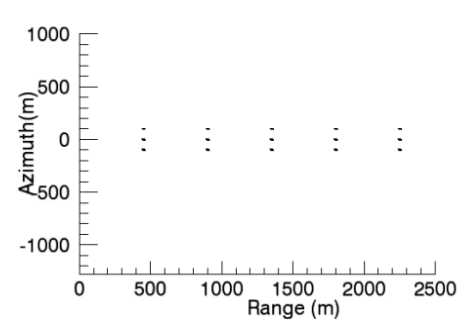

Fig.2 Simulated Scene

Table II SIMULATION PARAMETERS

\begin{tabular}{|c|c|}
\hline Stepped number & 4 \\
\hline Recombined bandwidth & $1.8 \mathrm{GHZ}$ \\
\hline Sub-bandwidth & $450 \mathrm{MHz}$ \\
\hline $\begin{array}{c}\text { Center frequency of the } \\
\text { recombined signal }\end{array}$ & $9.7 \mathrm{GHz}$ \\
\hline Velocity & $200 \mathrm{~m} / \mathrm{s}$ \\
\hline Azimuth resolution & $0.18 \mathrm{~m}$ \\
\hline
\end{tabular}

Original CSA and bandwidth segmentation method are applied to focus the received LFM signal respectively. Images will be depicted and compared in this section. With the simulation parameters, the focused scene size ${ }^{[23]}$ when restricting the maximum residual quadratic phase error to be less than $\frac{\pi}{2}$ is $380 \mathrm{~m}$. As depicted in Fig. 3, the simulated scene size in range dimension is $1800 \mathrm{~m}$, which is far beyond $380 \mathrm{~m}$. The impulse response function (IRF) of PT at scene center, PTs $750 \mathrm{~m}$ far from the scene center and $1500 \mathrm{~m}$ far from the scene center are depicted in Fig.4-6, respectively, which validate the bandwidth segmentation method obviously.

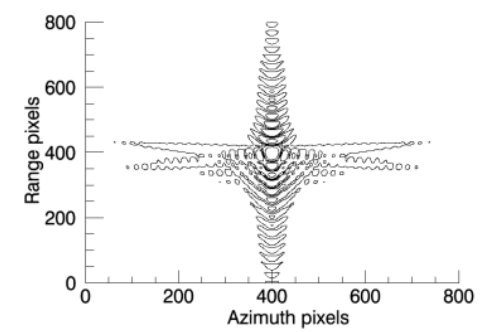

(a)

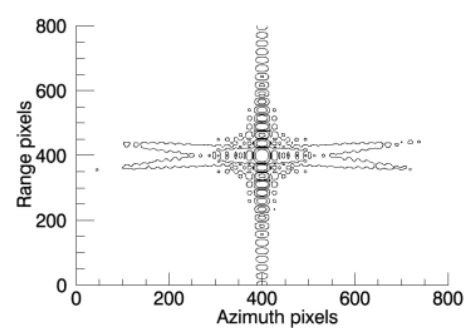

(b)

Fig. 3 IRFs of PT at the scene center. (a) CSA processing;(b) Bandwidth segmentation method ( 1 range pixel $=0.0032 \mathrm{~m}, 1$ azimuth pixel $=0.0073 \mathrm{~m}$ ). 


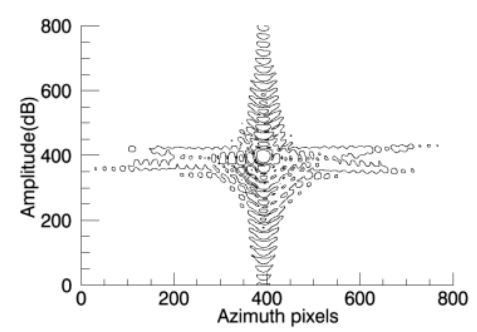

(a)

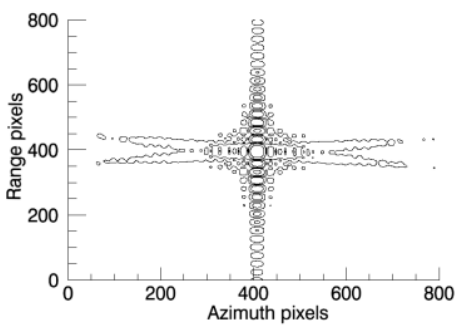

(b)

Fig. 4 IRFs of PT 750m far from the scene center. (a) CSA processing;(b) Bandwidth segmentation method (1 range pixel $=0.0032 \mathrm{~m}, 1$ azimuth pixel $=0.0073 \mathrm{~m}$ )

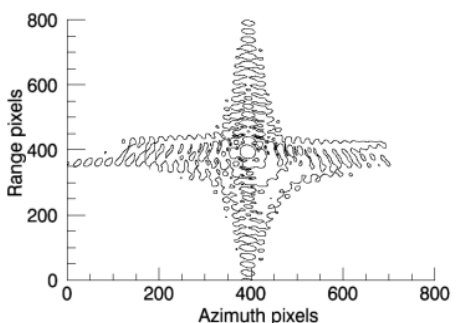

(a)

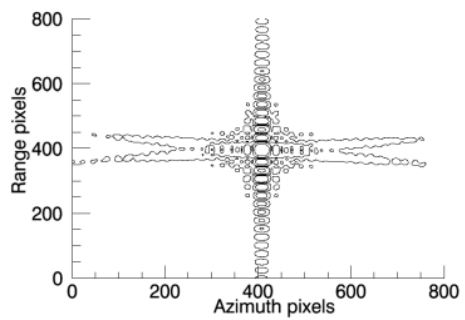

(b)

Fig. 5 IRFs of PT 1500m far from the scene center. (a) CSA processing;(b) Bandwidth segmentation method ( 1 range pixel $=0.0032 \mathrm{~m}, 1$ azimuth pixel $=0.0073 \mathrm{~m}$ )

\section{CONCLUSION}

In our study, a novel bandwidth segmentation methodology is proposed to process the received chirps in ultra-high resolution SAR. Performance analysis and PT simulation indicates that this processing method can improve the focal quality of SAR image and extend the focused scene size effectively.

\section{ACKNOWLEDGMENT}

We would like to thank the support of Jiangsu Natural Sc ience Foundation of BK2011757, Scientific Research Foundat ion of Nanjing University of Posts and Telecommunications of NY211037, NY210071 and NY212021.

\section{REFERENCES}

[1]. A. R. Brenner, J. H. G. Ender, "First experimental results achieved with the new very wideband SAR system PAMIR", Proceeding of EUSAR 2002, Germany VDE, 2002, pp. 81-86.

[2]. S. H. Lim, C. G. Hwang, S. Y. Kim and et.al, "Shifting MIMO SAR system for high-resolution wide-swath imaging “, Journal of Electromagnetic Waves and Applications, vol. 25, no. 8-9, 2011, pp. 1168-1178.

[3]. D. Reale, G. Fornaro, and et.al, "Tomography imaging and monitoring of buildings with very high resolution SAR data", IEEE Geoscience and Remote Sensing Letters, vol. 8, no.4, 2011, pp.661-665.

[4]. T. S. Lim, C. S. Lim, V. C. Koo, and et. al, "Autofocus algorithms performance evaluations using an integrated SAR product simulator and processor", Progress In Electromagnetics Research B, vol.3, 2009, pp. 316-329.

[5]. Y. K. Chan, V. C. Koo, "An introduction to synthetic aperture radar(SAR)", Progress In Electromagnetics Research B, vol.2, 2008, pp. 27-60.

[6]. Y. K. Chan, S. Y. Lim, "Synthetic aperture radar(SAR) signal generation", Progress In Electromagnetics Research B, vol.1, 2008, pp. 269-290.

[7]. M. Y. Chua and V. C. Koo, "FPGA-based chirp generator for high resolution UAV SAR", Progress In Electromagnetics Research, PIER 99, 2009, pp.71-88.

[8]. J. Mittermayer, A. Moreira, O. Loffeld, "Spotlight SAR data processing using the frequency scaling algorithm", IEEE Transactions on Geoscience and Remote Sensing, vol. 37, no. 5, 1999, pp. 2198-2214.

[9]. W. M. Brown, R. J. Fredricks, "Range-doppler imaging with motion through resolution cells", IEEE Transactions on Aerospace and Electronic Systems, vol. 5, no.1, 1969, pp.98102.

[10]. R. K. Raney, H. Rung, R. Bamler, I. G. Cumming, "Precision SAR processing using chirp scaling", IEEE Transaction on Geosciences and Remote Sensing, vol. 32, no. 4, 1994, pp. 786-799.

[11]. X. H. Mao, D.Y. Zhu, and Z.D. Zhu, "Signatures of moving target in polar format spotlight SAR image", Progress In Electromagnetics Research, PIER 92, 2009, pp. 47-64.

[12]. E. C. Gaugg, D. G. Long, "Generalized Frequency-Domain SAR Processing", IEEE Transactions on Geoscience and Remote Sensing, vol. 47, no.11, 2009, pp. 3761-3773.

[13]. T. Long, F. Li, and et.al, "Improved chirp scaling algorithm based on new integral kernel", IET Radar Sonar and Navigation, vol. 5, no. 4, 2011, pp. 466-472. 